\title{
Optimization of Gas Condensate Field Production Performance by Fault Block Modelling and Decision Under Uncertainty Technique
}

\author{
Ursin, Jann-Rune, Stavanger College \\ Zolothukin, Anatoly, Stavanger College
}

Paper presented at the $5^{\text {th }}$ European Conference on the Mathematics of Oil Recovery, Leoben, Austria, 3-6 Sept.1996

\begin{abstract}
Optimization of a field development scenario is strongly related to feasible evaluation of reservoir uncertainty. In case of faulted and highly compartmentalized reservoirs the degree of success in production performance forecast depends on how uncertainty, associated with the number of faults, their nature, location and effectiveness as flow barriers, is handled.

The Fault Block Model described in this paper is particularly developed for production from gas or gascondensate fields. The model redefines individual fault block traps in terms of block volume and cross-flow probability to the adjacent blocks, where geological uncertainties are carried through in the model by error propagation techniques. Three different realizations of the reservoir model are considered: one representing the most likely (average) reservoir where the two others represent the extreme alternatives, reflecting reservoir uncertainties. The fault block evaluation is treated as input to material balance calculation, where reservoir production is assumed to take place from individual blocks.
\end{abstract}

Dependency of the field performance on the uncertainty variation has also been validated by applying fussy set theory and its applications. A linear possibility regression model for a field performance has been obtained demonstrating directly how uncertainty in production evaluation is generated. As a result of this study it has been shown that the uncertainty in the reservoir characterization generated by faulting, together with the field development strategy have the greatest impact on the production performance from the entire reservoir.

\section{Introduction}

In any plan for development and production from oiland gas reservoirs, it is important to create a reliable and trustworthy picture of the uncertainties involved, and of their consequences for reserve estimation and production. Seen from a reservoir engineering point of view, this problem is commonly tackled by constructing and studying a mathematical simulation model which is often a direct interpretation of the reservoir geological model. Uncertainty related to production is then evaluated by parameter variation, where the model itself is not significantly changed.

In conditional simulation techniques [1], uncertainty in well production is evaluated through statistical representation of typical reservoir characteristics. In stochastic geological models [2] reservoir uncertainty is considered as distribution functions. Other methods such as decline-curve performance $[3,4]$, predict productions from multi-well pools through statistical well rate analysis.

For strongly faulted reservoirs where the uncertainty is particularly linked to the conception of the reservoir as such, other approaches should be explored. In such reservoirs, the consideration of faults as sealing barriers to fluid flow is highly exposed to misinterpretation. For such reservoirs the uncertainty is not only related to estimation of volumes in-situ, but also to the degree of communication within the reservoir. In such compartmentalized reservoirs, a more correct approach would be to explore the reservoir uncertainty by considering a multi-model approach, i.e. by investigating alternative models of the reservoir.

Modern reservoir simulation models, generated for the use of the finite-difference technique such as the Ec1

lipse program package, are results of extensive and often 
tedious work with respect to layering, griding, fault emplacement and local grid refinement. Due to their complexity, they are not easy to modify in order to visualize alternative possibilities of reservoir extension and compartmentalization. There is therefore a definitive need for simulation models which are easy to operate when vital reservoir characteristics are changed due to uncertainty in reservoir communication across faults, i.e. definition of block size or well drainage volume (confined reservoir volume where fluid flow is not restricted due to faults).

The Fault Block Model (FBM) $[5,18]$ has the abilities as mentioned above. The model is particularly useful for gas or gas-condensate reservoirs where the reservoir productivity is considered to be good, i.e. high gas mobility and low degree of stratigraphic layering as hindrance to flow.

The Fault Block Model is a multi-compartment model and multi-compartment models have previously been used for history matching and as a tool for detecting and qualifying compartmented reservoir structure $[6,7]$.

The model to be discussed in this paper has the ability to redefine the reservoir model in terms of blocks and communication between blocks. The model suggests where production wells should be drilled and in which sequence. Production volumes are evaluated using material balance calculations [8] and the uncertainty is addressed using error analysis.

Three different realizations of reservoir models are created; one representing the most likely (average) reservoir while two others represent the extreme alternatives, reflecting the reservoir uncertainties.

\section{The Fault Block Model}

\section{Model philosophy}

The model described in this paper is designed for production from gas or gas condensate fields, where modelling of highly faulted and compartmentalized reservoirs is severely impeded by the nature and extent of known and undetected faults and, in particular, their effectiveness as flow barriers.

The basic philosophy behind the FBM is that it is possible, in theory at least, to define a drainage volume, i.e. a part of the reservoir (a drainage block volume), from which a single well or a cluster of neighbouring wells, will produce the reserves contained in that block. This blocks will have imaginary boundaries with adjacent blocks which, eventually, could be produced by other wells.

If it would be possible to identify these block volumes (where the internal productivity is assumed to be good), we could estimate the production by material balance calculation. The question here is therefore not how production is evaluated but rather how the reservoir should be divided in connection with the number of producing wells and where to locate wells in the reservoir.

Error calculation is introduced through uncertainty in block volume and in cross-flow communication probability ( to be defined late). Uncertainty is carried through in the model by error propagation techniques.

The model suggests location and sequence of wells, based on a principle that production will be optimized when wells are located in blocks where the potential of drainage block volume (per well) is maximized (see proof in Appendix). This principle secures the production from those parts of the reservoir where the gas potentials are the highest.

\section{Model description (theory)}

We start this short description with some definitions:

Initial block volume has a geological reference and is defined as a physical volume of gas confined by faults which might act as a sealing barrier to fluid flow. The volume of gas contained in such a block is given by $V_{i}$.

Cross-flow communication probability is carrying information about the internal communication in the reservoir. The probability for communication between neighbouring blocks, across a borderline is $P_{i, j}$. When $P_{i, j}>0$, cross-flow exist and the fault is not a sealing barrier.

Drainage block volume is a virtual gas volume, a redefined block volume, where additional parts of volume from neighbouring blocks are added. This volume is given by,

$$
{ }^{1} V_{i}=\sum_{j}\left(P_{i, j} \cdot V_{j}\right),
$$

and contain parts of the gas contained in the neighbouring blocks.

In addition to the block volume and the cross-flow communication probability we also evaluate the uncertainty in the block volume, $\Delta V_{i}$ and the uncertainty in the cross-flow communication probability, $\Delta P_{i, j}$. The uncertainties above might be associated with the standard deviation or to a defined confidence level. (The uncertainty numbers are very important parameters in the analysis of error propagation, and finally in the definition of alternative production profiles.)

Let us consider a strongly faulted reservoir which has been compartmentalized into $\mathrm{N}$ number of blocks and let us assume that we have decided to produce the reserves using a fixed number of wells, were the number of wells could be larger than $\mathrm{N}$, if so happens.

The first step is to locate the block from where the first well will start production. This is done by evaluating the overestimated block volumes defined by Eq.1. 
The block containing the maximum part of the reserves is chosen, i.e. ${ }^{1} V_{m} \geq{ }^{1} V_{i}, \forall i=1, \ldots, N$, and the volume of block $m$ is defined by,

$$
{ }^{1} V_{m}=\sum_{j=1}^{N}\left(P_{m, j} \cdot V_{j}\right)
$$

where ${ }^{1} V_{m}$ is the first generation drainage block volume.

Before we can define the second largest block volume, we have to redefine the cross-flow communication probabilities for the remaining blocks. This step is necessary in order to correct for the overlapping of block volumes when the second largest block is to be defined. Cross-flow communication probability is redefined, and we write,

$$
{ }^{1} P_{i, j}=P_{i, j}-f(i)\left(P_{i, j} \cdot P_{m, j}\right), \quad i \neq m .
$$

$f(i)$ is the scaling factor, defining the relative partition of reserves when two wells are located in neighbouring blocks.

The second well is now located in the second largest block volume, ${ }^{2} V_{n} \geq{ }^{2} V_{i}, \forall i \neq m$, where $V_{i}$ is defined as,

$$
{ }^{2} V_{i}=\sum_{j=1}^{N}\left({ }^{1} P_{i, j} \cdot V_{j}\right), \quad j \neq m .
$$

Volume interaction for block $m$ between block $m$ and $n$ is ajusted by redefining the cross-flow communication probabilities for the $m$-th block,

$$
{ }^{1} P_{m, j}=P_{m, j}-f(m)\left(P_{m, j} \cdot{ }^{1} P_{n, j}\right) .
$$

The last step in the cycle, which are repated until all wells are located, redefines the cross-flow communication probability for the estimation of third largest block volume,

$$
{ }^{2} P_{i, j}={ }^{1} P_{i, j}-f(i)\left({ }^{1} P_{i, j} \cdot{ }^{1} P_{n, j}\right), \quad i \neq m, n
$$

The location of the third well and all consequtive wells are defined using Eq.2 to Eq.3, repeatingly.

Uncertainties are introduced in the drainage block volume by error propagation of the initial block volume uncertainty $\Delta V_{i}$ and the uncertainty in the initial crossflow communication probabilities $\Delta P_{i, j}$.

In order to illustrate this process, we may consider the drainage volumes after $k$ wells have been located. The drainage volumes are then defined,

$$
{ }^{k} V_{i}=\sum_{j=1}^{N}\left({ }^{k-1} P_{i, j} \cdot V_{j}\right)
$$

where the division of drainage volumes represents the most likely compartmentalization of the reservoir.
Due to the uncertainties $\Delta V_{i}$ and $\Delta P_{i, j}$, there exists an uncertainty $\Delta^{k} V_{i}$, for all drainage block volumes, defined by,

$$
\Delta^{k} V_{i}=\left\{\sum_{l}\left(\left[\Delta V_{i} \cdot P_{i, l}\right]^{2}+\left[V_{i} \cdot \Delta P_{i, l}\right]^{2}\right)\right\}^{1 / 2},
$$

where $P_{i, l}$ is summed over all generations from ${ }^{1} P_{i, j}$ to ${ }^{k-1} P_{i, l}$.

The error propagation in the cross-flow communication probabilities $P_{i, j}$ are defined as part of the process discribed by the equations, Eq.2 to Eq.3, and we write,

$$
\begin{aligned}
& \Delta P_{i, j}=\left\{\left[\Delta P_{i, j}\right]^{2}+\right. \\
& \left.f^{2}(i) \sum_{l}\left(\left[P_{i, j} \cdot \Delta P_{l, j}\right]^{2}+\left[P_{l, j} \cdot \Delta P_{i, j}\right]^{2}\right)\right\}^{1 / 2} .
\end{aligned}
$$

As a final result of the Fault Block Modelling process three sets of drainage block volume data are created. The most likely alternative, where gas is redistributed according to the definition of the cross-flow communication probabilities, defined by Eq.4. In addition we get a minimum - and a maximum value of the drainage block volume, represented by ${ }^{k} V_{i} \mp \Delta^{k} V_{i}$.

Finally material balance calculation is carried out in accordance to the specification given in the input file containing general reservoir data, production data, PVT data and data concerning the simulation process.

\section{Model example}

In order to demonstrate the process by which wells are located in different blocks, we have chosen as an example of a strongly faulted gas-condensate field. As a practical demonstration of the fault block modelling process, we focus first on a smaller region in this field, containing only five initial blocks were two wells are to be located. The region shown in Fig. 1 is partly isolated from the larger reservoir, seen in Fig.2.

In Fig.1, we have tried to illustrate the process in which parts of adjacent block volumes are added to the volume of the drainage block where the wells are located. For wells located in neighbouring blocks, the volume shared will be divided in equal parts and assigned to each of them( see equations above where $f(i)=1 / 2)$.

The probability of cross-flow communication between adjacent blocks are defined according to geological expectation and set to a number between 0 and 1 (where 0 means no communication across block boundary). Fig.1a) gives the block names and their corresponding cross-flow communication probability.

If we choose to produce the gas in this region, using only 2 wells, one well in block $B 2$ and one well in $B 4$,we would expect the gas in the other blocks to be partly assigned to these two drainage blocks. Based on statistical reasons, as described in the theory section, we may redistribute the gas in accordance to the probability of communication across block boundaries. The new 

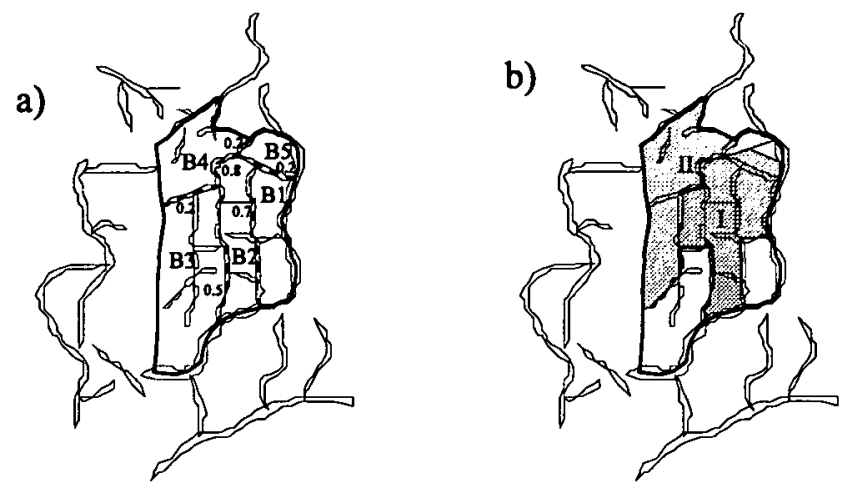

Figure 1: Redistribution of block volume in a regional field.

volumes formed are called the drainage volume $I$ and $I I$, from which we finally would produce the gas using material balance technique. Some parts of the reservoir gas, as seen in Fig.1b), are not assigned to either of the two drainage volumes. This gas will not be produced.

\section{Simulation study}

\section{Reservoir example}

Vairious simulation studies have been carried out using the gas-condensate reservoir as the appropriate example. This reservoir has been compartmentalized in 22 blocks, as shown in Fig.2. The reservoir example considered has good gas productivity with permeabilities larger than $100 \mathrm{mD}$. The combination of good reservoir productivity and the large number of major faults which are considered to be partly sealing, compartmentalizes the reservoir and makes an optimum gas recovery plan difficult to define. The aquifers connected to the reservoir are expected to be small and of minor importance for gas production.

In this study we have simulated the production of gas and condensate, using the Fault Block Model. The reservoir volume, summed over all 22 initial blocks (see Fig.2), contains $172.7 \mathrm{GSm}^{3}$ of gas initially in place. The reservoir has been drained using a maximum well rate of $2.14 \mathrm{MSm}^{3} /$ day and a plateau rate of 19.32 $\mathrm{MSm}^{3} /$ day. Initial reservoir pressure of 437 bar has been assigned to all blocks and production has been conducted with a minimum well head pressure of 50 bar. No gas lift tables have been used.

All together eight full field simulation runs have been accomplished with a maximum number of $11,13,15$, $17,19,21,23$ and 25 wells. It is believed that the optimum number of wells needed to produce this reservoir is contained in the range of cases simulated. In Fig. 3 we have compared the two extreme cases with 11 and 25 wells, respectively. The firgure shows the division of drainage block volume in the two cases. When the number of production wells are increased, less gas is generally contained in each drainage block volume since

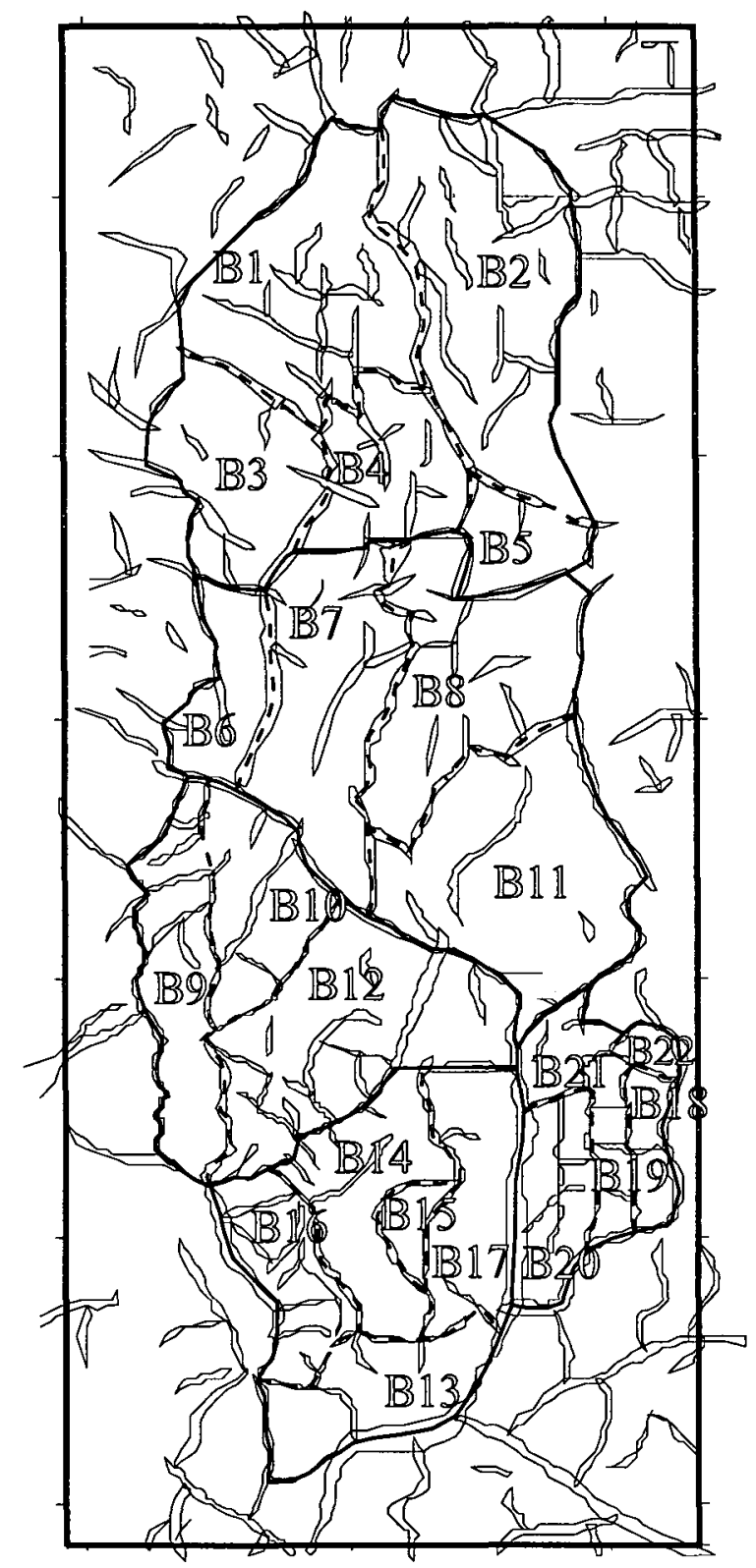

Figure 2: Reservoir subdivided into regions and blocks.

the number of drainage block volumes are increased. Fig.3 points directly to esential effect of the Fault Block Model where drainage block volumes are formed as a function of cross-flow communication probabilities and the number of wells allocated to gas production.

Total gas rate and production profile are shown in Fig.4. The figure shows a difference of about 5 years in prolonged plateau production when the number of wells is increased from 11 to 25 . The decline profile depends significantly on the number of wells, where fewer wells implicate longer decline period. With 25 wells in production, more reserves are contacted and more gas will be produced as compared to the case of 11 wells.

Given the appropriate parameters of capital costs, labour and other techincal expences, we could define an optimum development scenario for this field. In this 


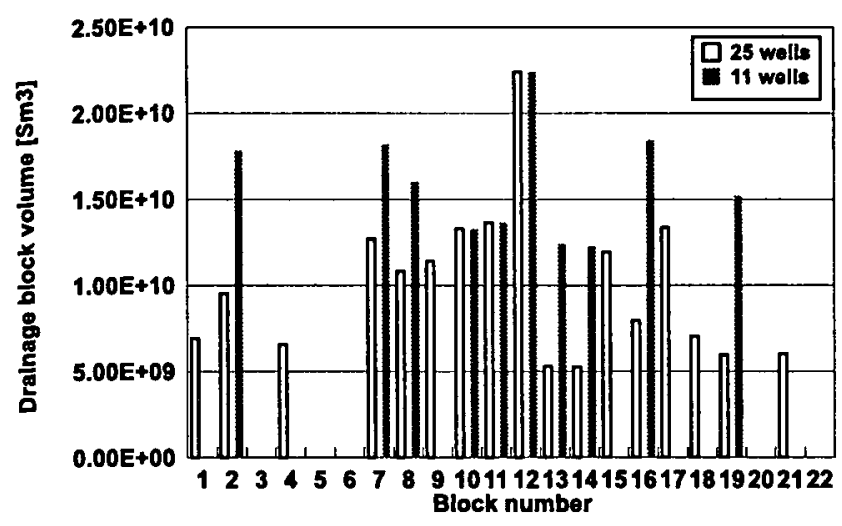

Figure 3: Drainage block volume as function of number of production wells.

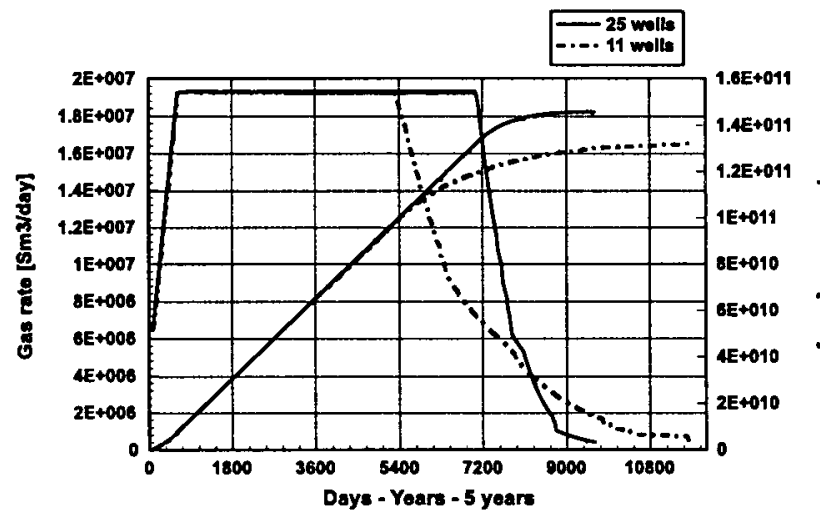

Figure 4: Gas rate and production profiles for two cases of 11 and 25 wells, respectively.

paper, however, we have chosen to focuse our attention to the uncertainty connected to the development of the entire field rather then on its optimization.

\section{Uncertainty in simulations}

The effect of uncertainty in cross-flow communication probability $\Delta P$, is to visualize alternative models of the reservoir. The two different models are characterized as,

a more opened reservoir, a less compartmentalized reservoir where communication across fault boundaries between blocks are more likely to be open to flow than in,

a more closed reservoir which represent a more pessimistic view with respect to internal communication within the reservoir.

Even though the drainage volumes are slightly changed in the two models, we maintain the same well locations and production start up time for all wells as in the "normal" model.

Uncertainty in block volume, $\Delta V$, will naturally have a direct impact on the gas production profiles. In the optimistic case, gas is added to the different blocks in accordance to the fault block process and in the pessimistic case, gas is subtracted.

In order to demonstrate the implications of uncertainty in cross-flow communication; $\Delta P$, and the uncertainty in block volume; $\Delta V$, we have assumed these uncertainties to be $20 \%$ in all cases. The effect of uncertaintiy in block volume is more pronounced as compared to the cross-flow communication probabilities, as could be expected when the two parameters are set to the same value.

We have taken the 25 well case as an example and in Fig.5, where we show the total gas rate and gas production for the optimistic- and the pessimistic case, respetively. The difference between the two pair of profiles demonstrate the uncertainty connected to the gas production. Similar profiles are found for the production of condnesate. From the figure we find the cumulative gas production to be about $30 \%$ higher in the optimistic case compared to the pessimistic case, and the plateau length is observed to be about 6 years longer in the optimistic case.

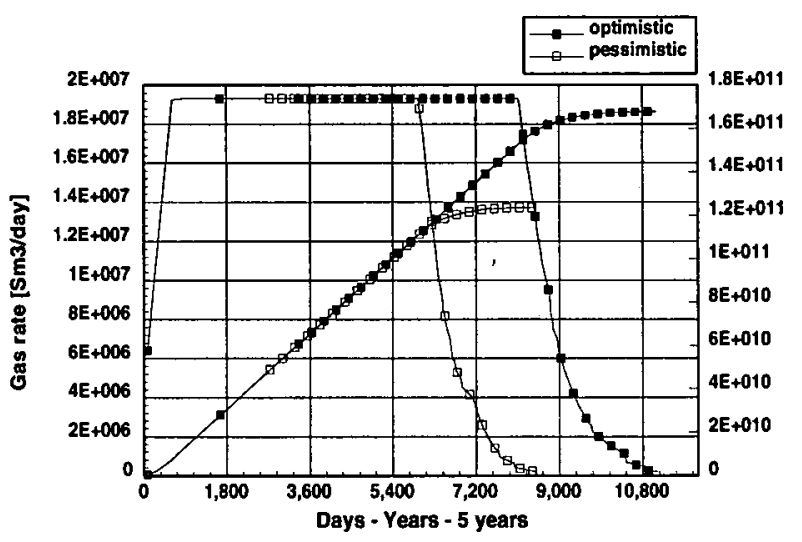

Figure 5: Alternative gas production profiles for 25 wells, where uncertainty in block volume and in crossflow communication probability are set to $20 \%$.

The difference in gas rate and in gas production is plotted in Fig.6. The difference between an optimistic and a pessimistic view on the in-place gas volume is about $45 \mathrm{GSm}^{3}$, more than 6 years of plateau production. The distribution shown in Fig.6 (open squares)gives a $\mathrm{FWHM}=29 \%$. Even though the uncertainty in block volume gives the most important contribution to the total uncertainty, the cross-flow communication probability part is more than about $5 \%$ of the total width of $29 \%$.

Fig.6, also demonstrates the effect of reduced reservoir uncertainty by decreasing the block volume uncertainty, $\Delta V$ from $20 \%$ to $10 \%$, for some central blocks in the reservoir. The reductiion is done for one block in each region in Fig.2. The effect is shown in Fig.6, where the FWHM is reduced from $29 \%$ to $22.5 \%$.

Using this option in the Fault Block Model we can locate where in the reservoir it would be most effective 


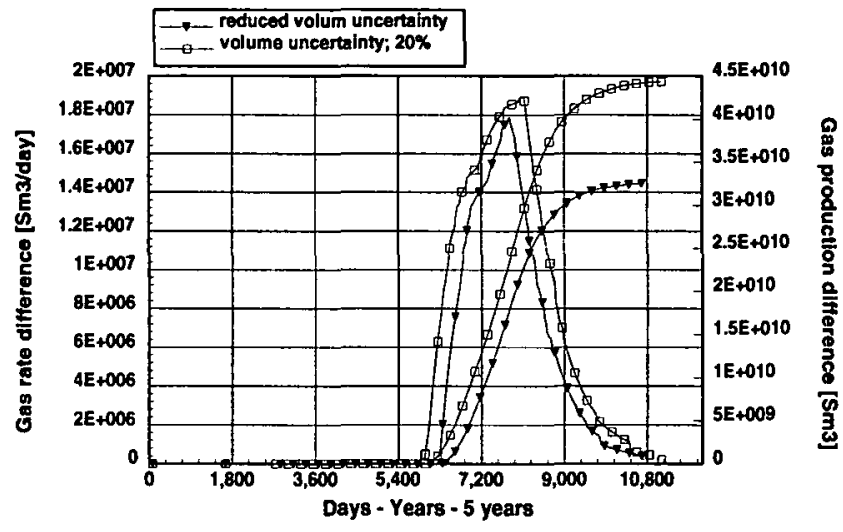

Figure 6: Gas rate and gas production difference when uncertainty in gas volume is $20 \%$ and when uncertainty is reduced for some blocks to $10 \%$.

Table 1: Pessimistic (PV), Most Likely (MLV) and Optimistic (OV) evaluations of cumulative gas production for different field development scenarious

\begin{tabular}{|c|c|c|c|c|}
\hline \multirow{2}{*}{ Scenario } & \multirow{2}{*}{$\begin{array}{c}\text { Number } \\
\text { of wells }\end{array}$} & \multicolumn{3}{|c|}{ Gas production, $10^{9} \mathrm{Sm}^{3}$} \\
\cline { 3 - 5 } & & PV & MLV & OV \\
\hline 1 & 11 & 74 & 97 & 114 \\
2 & 13 & 77 & 97 & 114 \\
3 & 15 & 91 & 111 & 130 \\
4 & 17 & 100 & 118 & 135 \\
5 & 19 & 102 & 122 & 140 \\
6 & 21 & 107 & 126 & 145 \\
7 & 23 & 111 & 130 & 149 \\
8 & 25 & 111 & 132 & 153 \\
\hline
\end{tabular}

to decrease the level of uncertainty in order to reduce an overall reservoir uncertainty. This study may take place as a sensitivity prior to start of production or as part of an optimization procedure parallel to normal production.

\section{Uncertainty analysis}

Let us try to examine uncertainties in production performance evaluation directly, i.e. by using fuzzy set theory [12], and, in particular, possibility theory [13] and fuzzy arithmetics [14]

Table 1 contains information about cumulative gas production for different field development scenarious evaluated by the FBM.

Let us assume that uncertainties in cumulative gas production can be represented by symmetrical fuzzy numbers (SFN) [15]. Symmetric triangular fuzzy number $A=(a, \alpha)$ is expressed by its most likely value $a$, and spread $\alpha$, as shown on the Fig. 7, a).

Introducing the level of presumption $h$ we can de- a)
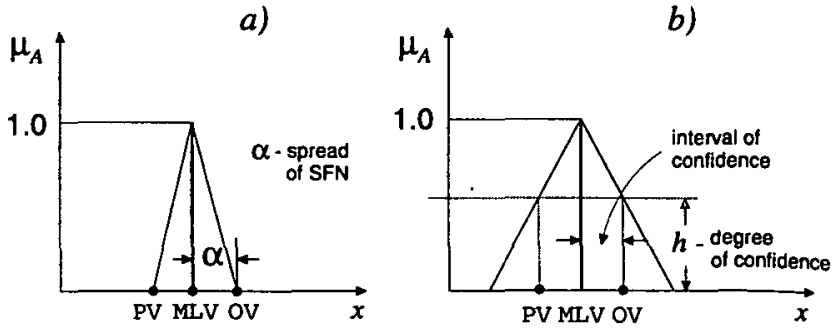

Figure 7: Symmetrical Fuzzy Numbers.

pict the interval of confidence $\left[P V^{(h)}, O V^{(h)}\right]$ containing pessimistic and optimistic evaluations at end points (Fig. 7, b).

Equation below shows a simple relationship between the spread $\alpha$ and the degree of confidence $h$ for a SFN:

$$
\left[P V^{(h)}, O V^{(h)}\right]=[M L V-\alpha(1-h), M L V+\alpha(1-h)]
$$

If pessimistic and optimistic evaluations are known then the spread of a SFN can be found at different levels of confidence. Note that the confidence is the strongest when $h=1$, and the weakest when $h=0$.

Let us assume that cumulative gas production depends on the total number of wells according to the following model:

$$
G_{p i}=A_{1}-\frac{A_{2}}{n_{i}}
$$

where $A_{1}$ and $A_{2}$ are SFNs and are constant, $n_{i}-$ number of wells in scenario $i$. Fuzzy numbers $A_{1}$ and $A_{2}$ can be found by means of a linear possibility regression model [16], which in our notation takes a form:

$$
\min _{\alpha} J\left(\alpha_{1}, \alpha_{2}\right)=\sum_{i=1}^{n}\left(\alpha_{1}+\frac{1}{n_{i}} \alpha_{2}\right)
$$

subject to the following constraints:

$$
\begin{array}{r}
G_{p i} \leq a_{1}-\frac{1}{n_{i}} a_{2}+(1-h)\left(\alpha_{1}+\frac{1}{n_{i}} \alpha_{2}\right) \\
G_{p i} \geq a_{1}-\frac{1}{n_{i}} a_{2}-(1-h)\left(\alpha_{1}+\frac{1}{n_{i}} \alpha_{2}\right) \\
a_{i} \geq 0 ; \quad \alpha_{i} \geq 0 ; \quad i \in[1, n s]
\end{array}
$$

where $n s$ is a number of scenarious considered.

Solution to this problem at two levels of confidence is shown below:

$\underline{h=0.5}$ :

$$
G_{p i}=(161.96,42.00)-\frac{749.1}{n_{i}}
$$

$\underline{h=0.67:}$

$$
G_{p i}=(161.96,63.64)-\frac{749.1}{n_{i}}
$$


It should be noted that using most likely values of the cumulative gas production and applying the Least Squares Method we derive at the following deterministic model:

$$
G_{p i}=164.67-\frac{811.92}{n_{i}}
$$

with its values being different from most likely values obtained by the Linear Possibility Regression Model.

Fig. 8 illustrates a linear possibility regression model for cumulative gas recovery against the total number of wells at 0.5 -level of confidence. As follows from the plot, all pessimistic and optimistic values for gas recovery are within the 0.5-interval of confidence, with its halfwidth equal to $21.0 \times 10^{9} \mathrm{Sm}^{3}$. This simple model can be further utilized in optimization of field deveopment scenario or long-term planning.

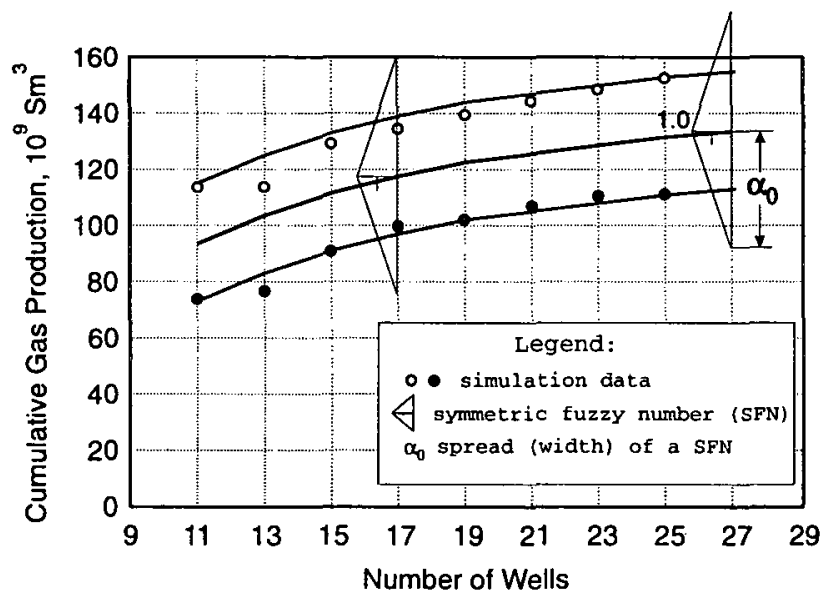

Figure 8: A linear possibility regression model for cumulative gas recovery vs total number of wells. (Cases with 11 and 13 wells were added after running the regression model.)

Fig. 9 shows how uncertainty in cumulative gas recovery evaluation depends on the total number of wells and the degree of presumption (level of confidence). As the trend indicates, evaluation of field performance developed by few wells creates large uncertainty in output data. By increasing the number of wells we reduce uncertainty in the recoverable reserves estimate. However, it might contradict with the optimal field development scenario. Therefore, there should be a compromize between the optimal value of recoverable reserves (or number of wells) and level of certainty at which this evaluation has been done.

Moreover, the degree of confidence also should be taken into consideration while analysing the data. Fig. 10 shows how uncertainty depends on the level of confidence. Too high level of confidence maintained in the input data creates too uncertain results. Again, there should be a compromize between these properties.

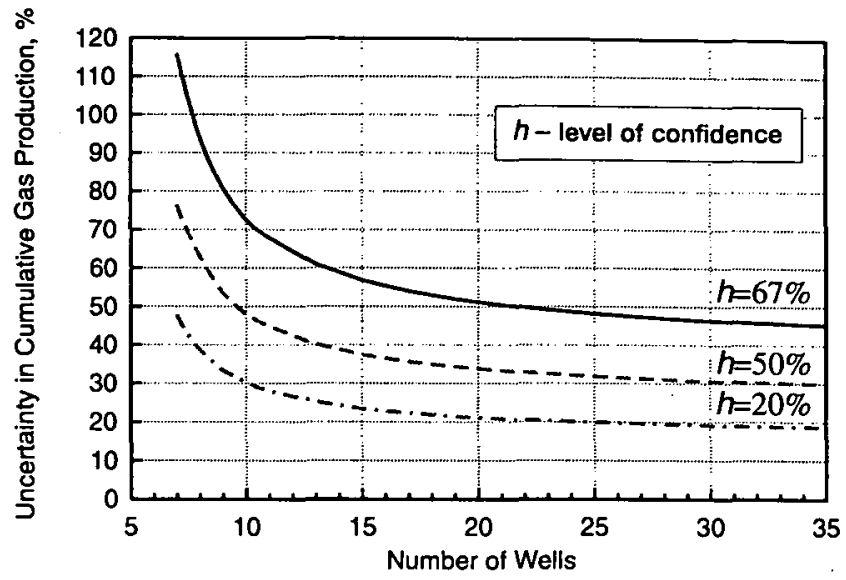

Figure 9: Uncertainty in cumulative gas production against the total number of wells. Several curves are associated with different levels of confidence.

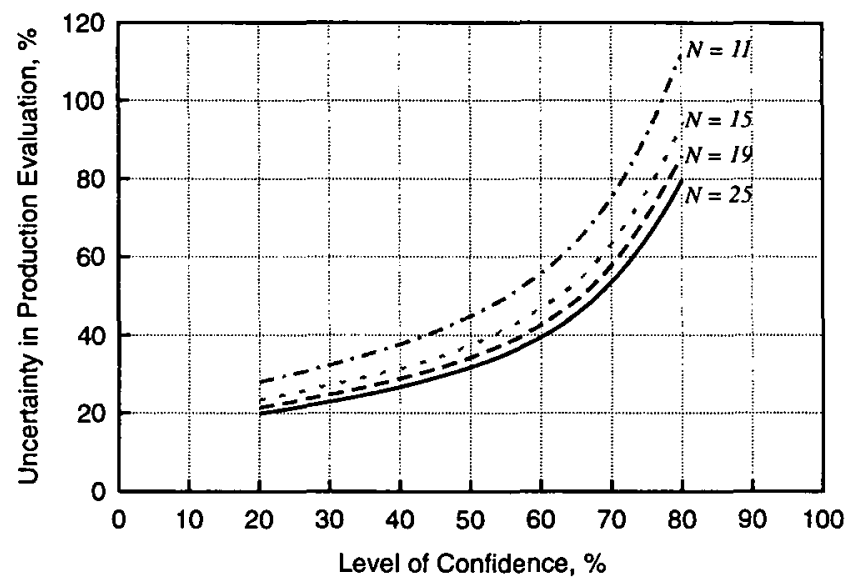

Figure 10: Uncertainty in cumulative gas production as a function of the level of confidence for different field development scenarious.

\section{Conclusions}

The Fault Block Model is designed to investigate the consequences of uncertainty in gas volume and the uncertainty connected to reservoir internal communication. The model recalculates the block volume in relation to communication probability across block boundaries. Uncertainty in block volume and uncertainty in cross-flow communication is calculated using error propagation techniques.

The Fault Block Model has been used in this paper, to study the implication of uncertainty introduced by block volume and by cross-flow communication probability. Several simulation cases have been studied where the number of wells are varied.

The Fault Block Model could be an important tool in revealing the effect of uncertainty in production form a strongly faulted gas reservoir, where the degree of compartmentalizing is a major source of reservoir uncertainty. 
Fault Block simulation could be used to optimize well location and to locate parts of the reservoir where reduced uncertainty will have the greatest impact on overall reservoir uncertainty.

A linear possibility regression model demonstrating the dependence of reservoir performance on the number of wells has been obtained. The model explicitely reveals uncertainty in the production performance and how it is connected to the uncertainty of the input data. this model can be easily applied to the optimisation of the field development strategy or to a long term planning.

\section{Nomenclature}

$V_{i} \quad$ block volume of gas.

$P_{i, j} \quad$ cross-flow communication probability.

$\Delta V_{i} \quad$ uncertainty in block volume.

$\Delta P_{i, j} \quad$ uncertainty in cross-flow communication probability.

$G_{p, i} \quad$ cumulative gas production.

$q_{i} \quad$ production rate.

$T_{i} \quad$ time required to drill and complete wells in the $i$-th block.

$t_{i} \quad$ duration of production from the $i$-the block. $N \quad$ number of blocks.

$n_{i} \quad$ number of wells in the $i$-th scenario of field development.

\section{References}

[1] K.B. Hird and M.G. Kelkar: "Conditional Simulation Method for Reservoir Description Using Spatial and Well-Performance Constraints", SPE Reservoir Engineering, May 1994.

[2] L.M. Meling, P.O. Mørkeseth and T. Langeland: "Production Forecasting for Gas Fields with Multiple Reservoirs", JPT, December 1990.

[3] R.A. Purvis: "Pool-Production and Well-Count Forecasts", Journal of Canadian Petroleum Technology, Nov.-Dec. 1990, Volume 29, No. 6

[4] D.C. Poon: "Decline Curves for Predicting Production Performance from Horizontal Wells", Journal of Canadian Petroleum Technology, Jan.Feb. 1991, Volume 30, No.1

[5] J.R. Ursin and P.O. Mørkeseth: "The Fault Block Model: A Novel Approach for Fauted Gas Reservoirs", Presented at the SPE International Conference \& Exhibition of Mexico held in Veracruze, Mexico, 10-13 October 1994, SPE 28732

[6] M.E. Lord and R.E. Collins: "Detecting Compartmented Gas Reservoirs Through Production Performance", Presented at the 66 th Annual Technical Conference and Exhibition of the Society of
Petroleum Engineers held in Dallas, TX. October 6-9, 1991, SPE 22941

[7] T.L. Hower and R.E. Collins: "Detecting Compartmentalization in Gas Reservoirs Through Production Performance", Presented at the 1989 Annual Technical Conference and Exhibition, San Antonio, October 8- 11, SPE 19790

[8] C.A. Ehlig-Economides: "Applications for $\mathrm{Mul}$ tiphase Compartmentalized Material Balance", Presented at the University of Tulsa Centennial Petroleum Engineering Symposium held in Tulsa, OK, U.S.A., 29-31 August, 1994, SPE 27999

[9] Annual Report, Norwegian Petroleum Directorate, 1992.

[10] A.M. Spencer and V.B. Larsen: "Fault Traps in the Northern North Sea", Geological Society Special Publication No 55, pp 281-298.

[11] J.R. Taylor: An Introduction to Error Analysis, Mill Walley, Calif. 1982,

[12] L.A. Zade: Fuzzy Sets: Information and Control, 1965, v.8, p.338-353.

[13] D. Dubois and H. Prade: Possibility Theory Plenum Press, New York, 1988, 263 p.

[14] A. Jones, A. Kaufmann, and H.-J. Zimmerman: Fuzzy Sets Theory and Applications, D. Reidel Publishing Company, Dordrecht, 1985.

[15] D. Dubois, H. Prade: Operations on Fuzzy Numbers, Int. J. Systems SCI, 1978, v. 9, p. 613-626.

[16] T. Terano, K. Asai, and M. Sugeno. Fuzzy Systems Theory and Its Applications, Academic Press, Inc., San Diego, 1992.

[17] A.I. Ermolaev: Models and optimization methods for designing automatic control systems, Moscow, 1991)

[18] J.R. Ursin and L. Mæland: "Multi Model Simulation Study for Strongly Faulted Gas Condensate Reservoir Using Fault Block Modelling", Presented at the Gas Technology Conference held in Calgary, Alberta, Canada, 28 April - 1 May 1996.

\section{Appendix}

Selection of a sequence of blocks development is related to the assignment problem which is a special case of an optimization problem under certainty when the objects (work, mashinery, agents, etc.) should be distributed among different tasks in such a way that no object can be divided among several tasks. 


\section{Different expected production rates from blocks}

In order to solve the problem let us suppose that the reservoir consists of $n$ blocks waiting for production and separated from each other by faulting. Assume also that production rate $q_{i}$ from each block is proportional to its initial resources. This assumption is in agreement with a statment that the drainage area per well is proportional to the size of a block, and a number of wells (and, thus, expected production rate from the block) is in direct proportion to its resources.

The main objective is to minimize the production loss from the idling blocks waiting to be put into production. If $c_{i j}$ denotes a loss of production from the $i$-th block that will be put into production in the $j$-th turn then an objective function can be written as [17]:

$$
f=\sum_{i=1}^{N} \sum_{j=1}^{N} c_{i j} y_{i j} \rightarrow \min _{y}
$$

where

$y_{i j}= \begin{cases}1, & \text { if the } i \text {-th block starts to produce in the } j \text {-th turn } \\ 0, & \text { otherwise }\end{cases}$

Other obvious conditions (constraints) are

$$
\begin{aligned}
& \sum_{i=1}^{N} y_{i j}=1 \\
& \sum_{j=1}^{N} y_{i j}=1
\end{aligned}
$$

The first equality means that simultaneous drilling and completion operations on several blocks is not possible. The second constraint says that each block can be put into production only once.

The problem (11)-(13) is referred to a linear problem of Boolean programming and can be solved by Hungarian method, method of branches, etc. Let us show a simple solution to this problem.

Suppose that the time required to drill and complete wells for each block is the same and equal $T$. Let us enumerate the blocks according to their expected production rates as shown below:

$$
q_{1} \geq q_{2} \geq \ldots \geq q_{N}
$$

Then the loss of production from the "idling" blockl $i$ that will be put into production in the $j$-th turn can be defined as

$$
c_{i j}=q_{i} T+(j-1) T q_{i}=j q_{i} T
$$

Substitution of ( 14 ) into ( 11 ) yields

$$
f=\sum_{i=1}^{N} \sum_{j=1}^{N} c_{i j} y_{i j}=T \sum_{i=1}^{N} q_{i} \sum_{j=1}^{N} j y_{i j} \rightarrow \min _{y}
$$

As follows from a constraint (13) the objective function $f$ can be reduced to the form:

$$
f=T \sum_{i=1}^{N} q_{i} j_{i} \rightarrow \min _{j_{i}}
$$

As can be shown the optimal solution is reached when $j_{i}=i$, i.e.

$$
f=T \sum_{i=1}^{N} q_{i} i
$$

\section{Different drilling and completion times}

Assume now that the wells are characterized by the same production rate $q$ but different drilling and completion times $T_{i}$.

Now the loss of production is evaluated as

$$
c_{i j}=q T_{i}+q \sum_{k=1}^{j-1} T_{k}
$$

and the objective function takes the form:

$$
\begin{array}{r}
f=\sum_{i=1}^{N} \sum_{j=1}^{N} c_{i j} y_{i j}=q \sum_{i=1}^{N} \sum_{j=1}^{N}\left(T_{i}+\sum_{k=1}^{j-1} T_{k}\right) y_{i j}= \\
q \sum_{i=1}^{N} \sum_{k=1}^{i} T_{k} \rightarrow \min _{T_{k}}
\end{array}
$$

which is equivalent to

$$
f_{1}=\sum_{i=1}^{N}[(N+1)-i] T_{i} \rightarrow \min _{T_{i}}
$$

and to

$$
f_{2}=\sum_{i=1}^{N} T_{i} \cdot i \rightarrow \max _{T_{i}}
$$

The optimum is reached when

$$
T_{1} \leq T_{2} \leq \ldots \leq T_{N}
$$

showing a sequence of putting blocks into production.

\section{Different $q_{i}$ and $T_{i}$}

Introducing a new variable $a_{i}=q_{i} / T_{i}$ we reduce this problem to the first case, i.e.

$$
\sum_{i=1}^{N} a_{i} i=\min f_{a_{i}}
$$

which is reached when the following conditions are satisfied:

$$
a_{1} \geq a_{2} \geq \ldots \geq a_{N}
$$

Another justification of the optimal strategy of putting blocks into production can be done by the following reasoning. 
Assume that an overall time of production from the entire field should be minimized. Then, denoting duration of production from the block $i$ as $t_{i}$ and assuming that the $i$-th block will start to produce in the $j$-th turn one can write the following optimization problem:

$$
f=\min _{j} \max _{i}\left\{T j+t_{i}\right\}
$$

If the blocks are enumerated in a descending order of duration times, i.e.

$$
t_{1} \geq t_{2} \geq \ldots \geq t_{N}
$$

then the optimal solution to the problem is

$$
T i+t_{i}
$$

which means that the block with the longest production time should be put into production first, the block with the second longest time - the second and so on. 